Stud. Univ. Babeş-Bolyai Math. 66(2021), No. 4, 723-738

DOI: 10.24193/subbmath.2021.4.10

\title{
Existence of positive solutions for a class of BVPs in Banach spaces
}

\author{
Lyna Benzenati, Svetlin Georgiev Georgiev and Karima Mebarki
}

\begin{abstract}
In this work, we use index fixed point theory for perturbation of expansive mappings by $\ell$-set contractions to study the existence of bounded positive solutions for a class of two-point boundary value problem (BVP) associated to second-order nonlinear differential equation on the positive half-line. The nonlinearity, which may exhibit a singularity at the origin, is written as a sum of two functions which behave differently. These functions, depend on the solution and its derivative, take values in a general Banach space and have at most polynomial growth. An example to illustrate the main results is given.
\end{abstract}

Mathematics Subject Classification (2010): 34B15, 34B18, 34B40, 47H08, 47H10.

Keywords: Boundary value problem, Green's function, unbounded interval, measure of noncompactness, fixed point index, sum operator.

\section{Introduction}

The theory of ordinary differential equations in Banach space is a rapidly growing area of research, it is developed for example in the books by Guo et al. [12], Guo and Lakshmikantham [11], Lakshmikantham and Leela [14], Deimling [2], and Zeidler [19] or in the papers by P. Li et al. [15] and by Y. Liu [16].

In the past decades, the study of BVPs defined on compact intervals has been considered by many authors with application of a huge variety of methods and techniques. However, BVPs defined on unbounded intervals are scarce, as they require other types of techniques to overcome the lack of compactness. Historically, these problems began at the end of nineteenth century with A. Kneser [13]. In this work, the lack of compactness is overcome with some techniques and specific tools.

Let $\mathcal{P}$ be a cone in some Banach space $E$, that is a closed convex subset such that $\alpha \mathcal{P} \subset \mathcal{P}$ for all positive real number $\alpha$ and $\mathcal{P} \cap(-\mathcal{P})=\{0\}$.

Notice that $E$ is partially ordered by cone $\mathcal{P}$, i.e. $x \leq y$ if and only if $y-x \in \mathcal{P}$. For details on cone theory see [11]. 
Throughout this paper, $(E,\|\cdot\|)$ denotes a Banach space and $\mathcal{P}$ is a cone in $E$. Being given a positive real parameter $k$ and $f: \mathbb{R}^{+} \times \mathcal{P} \times E \rightarrow \mathcal{P}$ a continuous function, we are interested in the study of the existence of bounded positive solutions to the second-order boundary value problem:

$$
\left\{\begin{array}{l}
-x^{\prime \prime}(t)+k^{2} x(t)=m(t) f\left(t, x(t), x^{\prime}(t)\right), \quad t \in(0,+\infty) . \\
x(0)=0, \lim _{t \rightarrow+\infty} x(t)=0
\end{array}\right.
$$

where the coefficient $m \in \mathcal{C}\left((0,+\infty), \mathbb{R}^{+}\right) \cap L^{1}\left((0,+\infty), \mathbb{R}^{+}\right)$may be singular at $t=0$ and it does not vanish identically on any subinterval of $(0,+\infty)$.

Also, we consider the problem

$$
\left\{\begin{aligned}
-y^{\prime \prime}+c y^{\prime}+\lambda y & =m(t) g\left(t, y(t), y^{\prime}(t)\right), \quad t \in(0,+\infty) \\
y(0)=\lim _{t \rightarrow+\infty} y(t) & =0
\end{aligned}\right.
$$

where $c, \lambda$ are positive constants and $g: \mathbb{R}^{+} \times \mathcal{P} \times E \rightarrow \mathcal{P}$ is a continuous function. Letting $k=\sqrt{\lambda+\frac{c^{2}}{4}}$ and $x(t)=y(t) e^{-\frac{c}{2} t}$, the problem (1.2) leads to the problem (1.1) for the new unknown $x$ and modified nonlinear term

$$
f\left(t, x(t), x^{\prime}(t)\right)=e^{\frac{-c}{2} t} g\left(t, e^{\frac{c}{2} t} x(t), e^{\frac{c}{2} t} x^{\prime}(t)+\frac{c}{2} e^{\frac{c}{2} t} x(t)\right) .
$$

Notice that the problems (1.1) and (1.2) arise in many applications in physics, combustion theory and epidemiology (see $[4,8,9,17,18]$ and the references therein). We will list some papers which provide a motivation for the introduction of this work. In [5], using the Krasnosels'kii fixed point theorem in cones for strict set-contractions, Djebali et al. investigated the existence of single and twin positive solutions to the following two-point boundary value problem of second-order nonlinear differential equations posed on the positive half-line:

$$
\left\{\begin{array}{l}
-x^{\prime \prime}(t)+k^{2} x(t)=m(t) f(t, x(t)), \quad t \in(0,+\infty) \\
x(0)=0, \lim _{t \rightarrow+\infty} x(t)=0
\end{array}\right.
$$

where the nonlinearity $f \in \mathcal{C}\left(\mathbb{R}^{+} \times \mathcal{P}, \mathcal{P}\right)$ satisfies a general polynomial growth condition. Motivated by the results obtained in the scalar case $E=\mathbb{R}$ in [7], the main purpose of this work is to discuss some existence results for the problem as that of [5], when $f$ depends also on the derivative. For this purpose, we employ the generalized fixed point index for the sum of an expansive mapping and a $\ell$-set contraction developed by Djebali and Mebarki in [6].

Now we describe in more details the structure of this work. This paper is devised in three sections. The first one is devoted to the preliminaries, recalling some basic concepts, and developing a new non compactness result that is needed for our purposes. The main results are presented in section 2 . We conclude with an example of application in section 3 . 


\section{Preliminaries}

\subsection{Measure of noncompactness and set-contraction}

In this paper, the concept of set contraction is related to Kuratowski's measure of noncompactness ( $\alpha$-MNC for short) $[3,10]$. Recall that the Kuratowski measure of noncompactness $\alpha(V)$ of a bounded subset $V$ of a Banach space $E$ is the infimum of positive numbers $\delta$ such that there exist finitely many sets of diameter at most $\delta$ which cover $V$.

Let $J \subset \mathbb{R}^{+}$. The Kuratowski measures of noncompactness of a bounded set in the spaces $E, \mathcal{C}(J, E), \mathcal{C}^{1}(J, E)$ and $X$ are denoted by $\alpha_{E}(),. \alpha_{\mathcal{C}}(),. \alpha_{\mathcal{C}^{1}}($.$) and \alpha_{X}($. respectively.

The following known results are used in this work.

Lemma 2.1. [10, Theorem 1.2.6]. Let $J=[a, b]$. If $H \subset \mathcal{C}^{1}(J, E)$ is bounded, $H$ and $H^{\prime}$ are equi-continuous, then

$$
\alpha_{\mathcal{C}^{1}}(H)=\max \left(\sup _{t \in J} \alpha_{E}(H(t)), \sup _{t \in J} \alpha_{E}\left(H^{\prime}(t)\right)\right),
$$

where $H(t)=\{u(t) \mid u \in H\}, t \in J$.

Lemma 2.2. [10, Theorem 1.2.2] If $H \subset \mathcal{C}(J, E)$ is bounded and equicontinuous, then $\alpha(H()$.$) is continuous on J$,

$$
\alpha_{\mathcal{C}(J, E)}(H)=\sup _{t \in J} \alpha(H(t)),
$$

and

$$
\alpha\left(\int_{J} x(t) d t \mid x \in H\right) \leq \int_{J} \alpha(H(t)) d t,
$$

Let $A: D \subset E \rightarrow E$ be a continuous operator. The operator $A$ is said to be bounded if it maps bounded sets into bounded sets, completely continuous if it maps bounded sets into relatively compact sets, and compact if the set $A(D)$ is relatively compact. The operator $A$ is said to be a $\ell$-set contraction, for some number $\ell \geq 0$, if it is bounded and $\alpha(A(V)) \leq \ell \alpha(V)$ for every bounded set $V \subset D$. If $\ell<1$, we say that $A$ is a strict set contraction.

We finish this part by giving the definition of an expansive mapping, let $(X, d)$ is a metric space. A mapping $T: D \subset X \rightarrow X$ is said to be expansive if there exists a constant $h>1$ such that

$$
d(T x, T y) \geq h d(x, y) \text { for all } x, y \in D .
$$

\subsection{The Green's function}

The following lemmas are concerned with the linear problem associated to (1.1). They provide useful estimates of the kernel $G$ and their proofs are omitted.

Lemma 2.3. Let $v$ be a function such that $v \in \mathcal{C}((0,+\infty), E)$ and $\int_{0}^{+\infty}\|v(t)\| d t$ exists. Then the problem

$$
\left\{\begin{array}{l}
-x^{\prime \prime}(t)+k^{2} x(t)=v(t), \quad t \in(0,+\infty) \\
x(0)=0, \lim _{t \rightarrow+\infty} x(t)=0
\end{array}\right.
$$


has a unique solution $x$ given by

$$
x(t)=\int_{0}^{+\infty} G(t, s) v(s) d s,
$$

where $G$ is the Green function of the problem, namely

$$
G(t, s)=\frac{1}{2 k}\left\{\begin{array}{lll}
e^{-k s}\left(e^{k t}-e^{-k t}\right), & \text { if } \quad 0 \leq t \leq s<\infty, \\
e^{-k t}\left(e^{k s}-e^{-k s}\right), & \text { if } \quad 0 \leq s \leq t<\infty .
\end{array}\right.
$$

Throughout this work, $0<\gamma<\delta$ will denote some fixed numbers. The interval $[\gamma, \delta]$ will play a key role in estimating the solutions of the problem (1.1). Let

$$
\begin{aligned}
\Lambda_{0} & =\min \left(e^{-k \delta}, e^{k \gamma}-e^{-k \gamma}\right) \\
\Lambda_{1} & =\min \left(\frac{1-k}{1+k} e^{-k \delta}, e^{k \gamma}+\frac{k-1}{k+1} e^{-k \gamma}\right) \\
\Lambda_{2} & =\frac{k}{k+1} e^{-k \delta}
\end{aligned}
$$

Obviously, these constants are less than 1. Some fundamental properties of the kernel $G$ are given hereafter. The proofs are omitted.

Lemma 2.4. The Green's function $G$ satisfies the following estimates:

(a) $G(t, s) \geq 0, \quad \forall t, s \in \mathbb{R}^{+}$.

(b) $G(t, s) \leq G(s, s) \leq \frac{1}{2 k}, \quad \forall t, s \in \mathbb{R}^{+}$.

(c) $G(t, s) e^{-\mu t} \leq G(s, s) e^{-k s}, \quad \forall t, s \in \mathbb{R}^{+}, \forall \mu \geq k$.

(d) $G(t, s) \geq \Lambda_{0} G(s, s) e^{-k s}, \quad \forall t \in[\gamma, \delta], \forall s \in \mathbb{R}^{+}$.

Remark 2.5. The problem (1.2) is equivalent to the integral equation:

$$
y(x)=\int_{0}^{+\infty} e^{\frac{c}{2}(x-s)} m(s) G(x, s) f\left(s, y(s), y^{\prime}(s)\right) d s .
$$

The boundary conditions $y(0)=y(+\infty)=0$ follow from $G(0, s)=0, \forall s \geq 0$, and $\lim _{x \rightarrow+\infty} e^{\frac{c}{2} x} G(x, s)=0, \forall s \geq 0$, since $k>\frac{c}{2}$, where $G$ is given by $(2.1)$.

To show our existence results we will use the following lemma which contains some recent results of the fixed point index theory on the cones of Banach spaces for the sum of two operators (see [6]). Let $X$ be a real Banach space and $\mathcal{K} \subset X$ a cone.

Lemma 2.6. Let $U$ be a bounded open subset of $\mathcal{K}$ and $W$ be a subset of $\mathcal{K}$ such that $0 \in U \cap W$. Assume that $T: W \rightarrow E$ is an expansive mapping with constant $h>1$, $F: \bar{U} \rightarrow E$ be a $\ell$-set contraction with $0 \leq \ell<h-1$, and $F(\bar{U}) \subset(I-T)(W)$. Thus we have the following: if

$$
\|F x+T 0\| \leq(h-1)\|x\| \text { and } T x+F x \neq x \text { for all } x \in \partial U \cap W,
$$

then $i(T+F, U \cap W, \mathcal{K})=1$. 


\section{Main results}

We begin by a new representation formula for the measure of noncompact- ness in the space $X$.

Let $p: \mathbb{R}^{+} \rightarrow(0,+\infty)$ be a continuous function. Denote by $X$ the space consisting of all weighted functions $y$, continuously differentiable on $\mathbb{R}^{+}$which satisfy

$$
\sup _{x \in \mathbb{R}^{+}}\left(\left[\|y(x)\|+\left\|y^{\prime}(x)\right\|\right] p(x)\right)<\infty .
$$

Equipped with a Bielecki's type norm $\|y\|_{p}=\sup _{x \in \mathbb{R}^{+}}\left(\left[\|y(x)\|+\left\|y^{\prime}(x)\right\|\right] p(x)\right)$, it is a Banach space.

In the following, we develop a new non-compactness result in order to use it to show that an operator is $\ell$-set contraction in the space $X$.

Lemma 3.1. Let $B \subset X$ be such that the functions belonging in the sets

$$
\begin{aligned}
& p B=\{z \mid z(t)=y(t) p(t), y \in B\}, \\
& p B^{\prime}=\left\{z \mid z(t)=y^{\prime}(t) p(t), y \in B\right\},
\end{aligned}
$$

are almost equicontinuous on $\mathbb{R}^{+}$and $B$ is a bounded set in the sense of the norm

$$
\|y\|_{q}=\sup _{x \in \mathbb{R}^{+}}\left(\left[\|y(t)\|+\left\|y^{\prime}(t)\right\|\right] q(t)\right)
$$

where the function $q$ is positive, continuous on $\mathbb{R}^{+}$and satisfies

$$
\lim _{t \rightarrow+\infty} \frac{p(t)}{q(t)}=0
$$

Then

$$
\alpha_{X}(B)=\max \left(\sup _{t \in \mathbb{R}^{+}} \alpha_{E}(B(t) p(t)), \sup _{t \in \mathbb{R}^{+}} \alpha_{E}\left((B)^{\prime}(t) p(t)\right)\right),
$$

where $B(t)=\{u(t) \mid u \in B\}$ for $t \in \mathbb{R}^{+}$.

Proof. Let $B \subset X$ be bounded in the sense of the norm

$$
\|y\|_{q}=\sup _{t \in \mathbb{R}^{+}}\left(\left[\|y(t)\|+\left\|y^{\prime}(t)\right\|\right] q(t)\right) .
$$

Thus there exists $r>0$ such that $\|y\|_{q} \leq r$ for all $y \in B$. Since the function $q$ is positive on $\mathbb{R}^{+}$and satisfies $\lim _{t \rightarrow+\infty} \frac{p(t)}{q(t)}=0$, for any $\varepsilon>0$, there exists $T>0$ such that

$$
\begin{aligned}
& \left\|y\left(t_{1}\right) p\left(t_{1}\right)-y\left(t_{2}\right) p\left(t_{2}\right)\right\| \\
\leq & \frac{p\left(t_{1}\right)}{q\left(t_{1}\right)}\left\|y\left(t_{1}\right)\right\| q\left(t_{1}\right)+\frac{p\left(t_{2}\right)}{q\left(t_{2}\right)}\left\|y\left(t_{2}\right)\right\| q\left(t_{2}\right) \\
\leq & \frac{p\left(t_{1}\right)}{q\left(t_{1}\right)}\left(\left\|y\left(t_{1}\right)\right\|+\left\|y^{\prime}\left(t_{1}\right)\right\|\right) q\left(t_{1}\right)+\frac{p\left(t_{2}\right)}{q\left(t_{2}\right)}\left(\left\|y\left(t_{2}\right)\right\|+\left\|y^{\prime}\left(t_{2}\right)\right\|\right) q\left(t_{2}\right)<\varepsilon,
\end{aligned}
$$

and

$$
\begin{aligned}
& \left\|y^{\prime}\left(t_{1}\right) p\left(t_{1}\right)-y^{\prime}\left(t_{2}\right) p\left(t_{2}\right)\right\| \\
\leq & \frac{p\left(t_{1}\right)}{q\left(t_{1}\right)}\left\|y^{\prime}\left(t_{1}\right)\right\| q\left(t_{1}\right)+\frac{p\left(t_{2}\right)}{q\left(t_{2}\right)}\left\|y^{\prime}\left(t_{2}\right)\right\| q\left(t_{2}\right) \\
\leq & \frac{p\left(t_{1}\right)}{q\left(t_{1}\right)}\left(\left\|y\left(t_{1}\right)\right\|+\left\|y^{\prime}\left(t_{1}\right)\right\|\right) q\left(t_{1}\right)+\frac{p\left(t_{2}\right)}{q\left(t_{2}\right)}\left(\left\|y\left(t_{2}\right)\right\|+\left\|y^{\prime}\left(t_{2}\right)\right\|\right) q\left(t_{2}\right)<\varepsilon,
\end{aligned}
$$

uniformly with respect to $y \in B$ as $t_{1}, t_{2} \geq T$. 
We first claim that

$$
\alpha_{X}(B) \leq \max \left(\sup _{t \in \mathbb{R}^{+}} \alpha_{E}(B(t) p(t)), \sup _{t \in \mathbb{R}^{+}} \alpha_{E}\left(B^{\prime}(t) p(t)\right)\right) .
$$

Denote by $\left.B\right|_{[0, T]}$ and $\left.B^{\prime}\right|_{[0, T]}$ the restriction of $B$ and $B^{\prime}$ on $[0, T]$. Since the sets $B(t) p(t)$ and $B^{\prime}(t) p(t)$ are equi-continuous on $[0, T]$, Lemma 2.1 ensures that

$$
\begin{aligned}
\alpha_{\mathcal{C}^{1}}\left(\left.B p\right|_{[0, T]}\right) & =\max \left(\sup _{t \in[0, T]} \alpha_{E}(B(t) p(t)), \sup _{t \in[0, T]} \alpha_{E}\left(B^{\prime}(t) p(t)\right)\right) \\
& \leq \max \left(\sup _{t \in \mathbb{R}^{+}} \alpha_{E}(B(t) p(t)), \sup _{t \in \mathbb{R}^{+}} \alpha_{E}\left(B^{\prime}(t) p(t)\right)\right),
\end{aligned}
$$

where $\left.B p\right|_{[0, T]}=\{y(t) p(t): y \in B, t \in[0, T]\}$.

By the definition of the MNC $\alpha_{\mathcal{C}^{1}}$, there exists $\left\{B_{i}\right\}_{i=1}^{n}$ such that $B=\bigcup_{i=1}^{n} B_{i}$ and for $i=1, \ldots, n$,

$$
\operatorname{diam}_{\mathcal{C}^{1}}\left(\left.B_{i} p\right|_{[0, T]}\right) \leq \max \left(\sup _{t \in \mathbb{R}^{+}} \alpha_{E}(B(t) p(t)), \sup _{t \in \mathbb{R}^{+}} \alpha_{E}\left(B^{\prime}(t) p(t)\right)\right)+\varepsilon,
$$

where $\operatorname{diam}_{\mathcal{C}^{1}}($.$) denotes the diameter of the bounded subsets of \mathcal{C}^{1}([0, T], E)$.

Furthermore, for $i=1, \ldots, n$, fixed, for all $y_{1}, y_{2} \in B_{i}$ and $t \geq T$, we deduce from (3.2)-(3.4), for $i=1, \ldots, n$ the following estimates:

$$
\begin{aligned}
& \left\|\left(y_{1}(t)-y_{2}(t)\right)\right\| p(t) \\
\leq & \left(\left\|\left(y_{1}(t)+y_{1}^{\prime}(t)\right)\right\|+\left\|\left(y_{1}^{\prime}(t)-y_{2}^{\prime}(t)\right)\right\|+\left\|\left(y_{2}(t)+y_{2}^{\prime}(t)\right)\right\|\right) p(t) \\
\leq \quad & \left(\left\|\left(y_{1}(t)+y_{1}^{\prime}(t)\right)\right\| q(t)+\left\|\left(y_{2}(t)+y_{2}^{\prime}(t)\right)\right\| q(t)\right) \frac{p(t)}{q(t)}+\left\|\left(y_{1}^{\prime}(t)-y_{2}^{\prime}(t)\right)\right\| p(t) \\
\leq \quad & \varepsilon+\left\|y_{1}^{\prime}(t) p(t)-y_{1}^{\prime}(T) p(T)\right\|+\left\|y_{1}^{\prime}(T) p(T)-y_{2}^{\prime}(T) p(T)\right\| \\
& \quad+\left\|y_{2}^{\prime}(T) p(T)-y_{2}^{\prime}(t) p(t)\right\| \\
\leq & 2 \varepsilon+\max \left(\sup _{t \in \mathbb{R}^{+}} \alpha_{E}(B(t) p(t)), \sup _{t \in \mathbb{R}^{+}} \alpha_{E}\left(B^{\prime}(t) p(t)\right)\right)+\varepsilon+\varepsilon,
\end{aligned}
$$

and

$$
\begin{aligned}
& \left\|\left(y_{1}^{\prime}(t)-y_{2}^{\prime}(t)\right)\right\| p(t) \\
\leq & \left(\left\|\left(y_{1}(t)+y_{1}^{\prime}(t)\right)\right\|+\left\|\left(y_{1}(t)-y_{2}(t)\right)\right\|+\left\|\left(y_{2}(t)+y_{2}^{\prime}(t)\right)\right\|\right) p(t) \\
\leq \quad & \left(\left\|\left(y_{1}(t)+y_{1}^{\prime}(t)\right)\right\| q(t)+\left\|\left(y_{2}(t)+y_{2}^{\prime}(t)\right)\right\| q(t)\right) \frac{p(t)}{q(t)}+\left\|\left(y_{1}(t)-y_{2}(t)\right)\right\| p(t) \\
\leq \quad & \varepsilon+\left\|y_{1}(t) p(t)-y_{1}(T) p(T)\right\|+\left\|y_{1}(T) p(T)-y_{2}(T) p(T)\right\| \\
& +\left\|y_{2}(T) p(T)-y_{2}(t) p(t)\right\| \\
\leq \quad & 2 \varepsilon+\max \left(\sup _{t \in \mathbb{R}^{+}} \alpha_{E}(B(t) p(t)), \sup _{t \in \mathbb{R}^{+}} \alpha_{E}\left(B^{\prime}(t) p(t)\right)\right)+\varepsilon+\varepsilon .
\end{aligned}
$$

Therefore (3.4), (3.5) and (3.6) guarantee that

$$
\operatorname{diam}_{X}\left(B_{i}\right) \leq \max \left(\sup _{t \in \mathbb{R}^{+}} \alpha_{E}(B(t) p(t)), \sup _{t \in \mathbb{R}^{+}} \alpha_{E}\left(B^{\prime}(t) p(t)\right)\right)+4 \varepsilon
$$


Noting that $B=\bigcup_{i=1}^{n} B_{i}$, we infer

$$
\alpha_{X}(B) \leq \max \left(\sup _{t \in \mathbb{R}^{+}} \alpha_{E}(B(t) p(t)), \sup _{t \in \mathbb{R}^{+}} \alpha_{E}\left(B^{\prime}(t) p(t)\right)\right)+4 \varepsilon,
$$

and $\varepsilon$ being arbitrary, we deduce that

$$
\alpha_{X}(B) \leq \max \left(\sup _{t \in \mathbb{R}^{+}} \alpha_{E}(B(t) p(t)), \sup _{t \in \mathbb{R}^{+}} \alpha_{E}\left(B^{\prime}(t) p(t)\right)\right) .
$$

Conversely, we prove that $\max \left(\sup _{t \in \mathbb{R}^{+}} \alpha_{E}(B(t) p(t)), \sup _{t \in \mathbb{R}^{+}} \alpha_{E}\left(B^{\prime}(t) p(t)\right)\right) \leq \alpha_{X}(B)$.

Given $\varepsilon>0$, there exists $\left\{B_{i}\right\}_{i=1}^{n}$ such that $B=\bigcup_{i=1}^{n} B_{i}$ and $\operatorname{diam}_{X}\left(B_{i}\right) \leq \alpha_{X}(B)+\varepsilon$. Thus, for fixed $i$, for every $t \in \mathbb{R}^{+}$and all $y_{1}, y_{2} \in B_{i}$, we have

$$
\left\|\left(y_{1}(t)-y_{2}(t)\right)\right\| p(t)\|\leq\| y_{1}-y_{2} \|_{\omega}<\alpha_{X}(B)+\varepsilon
$$

and

$$
\left\|\left(y_{1}^{\prime}(t)-y_{2}^{\prime}(t)\right)\right\| p(t)\|\leq\| y_{1}-y_{2} \|_{\omega}<\alpha_{X}(B)+\varepsilon .
$$

Since $B(t)=\bigcup_{i=1}^{n} B_{i}(t)$, we have $\alpha_{E}(B(t) p(t)) \leq \alpha_{X}(B)+\varepsilon$.

Now $\varepsilon$ being arbitrary, we deduce that $\sup _{t \in \mathbb{R}^{+}} \alpha_{E}(B(t) p(t)) \leq \alpha_{X}(B)$.

In accordance with $B^{\prime}(t)=\bigcup_{i=1}^{n} B_{i}^{\prime}(t)$, we get $\sup _{t \in \mathbb{R}^{+}} \alpha_{E}\left(B^{\prime}(t) p(t)\right) \leq \alpha_{X}(B)$, where $H^{\prime}(t)=\left\{y^{\prime}(t) \mid y \in H\right\}, t \in \mathbb{R}^{+}$, whence the reversed inequality and then the desired result.

Let $\omega>0$ be a given real parameter. Consider the Banach space with weight function $e^{-\omega t}$

$$
X=\left\{x \in \mathcal{C}^{1}\left(\mathbb{R}^{+}, E\right): \sup _{t \in \mathbb{R}^{+}}\left(\left(\|x(t)\|+\left\|x^{\prime}(t)\right\|\right) e^{-\omega t}\right)<\infty\right\},
$$

endowed with the norm

$$
\|x\|_{\omega}=\sup _{t \in \mathbb{R}^{+}}\left(\left(\|x(t)\|+\left\|x^{\prime}(t)\right\|\right) e^{-\omega t}\right) .
$$

Define the cone

$$
\widetilde{\mathcal{K}}=\left\{x \in X: x \geq 0 \quad \text { on } \quad \mathbb{R}^{+}\right\} .
$$

With $\mathcal{K}$ we denote the set of all equi-continuous families in $\widetilde{\mathcal{K}}$.

Take $\varepsilon \in(0,1)$ and $p, q>0$ arbitrarily. Let $A, B_{1}, B_{2}, B_{3}, R, \tau$ be positive constants such that

$$
G(t, s)+\left|G_{t}(t, s)\right| \leq A, \quad t, s \in[0, \infty),
$$

and

$$
0<\tau<\frac{1}{4}, \quad A\left(B_{1}+B_{2} R^{p}+B_{3} R^{q}\right)<\min \{\tau, R\} .
$$

Define the conical shell

$$
\mathcal{K}_{R}=\left\{x \in \mathcal{K}:\|x\|_{\omega}<R\right\}
$$


Assume that

(H1). $f \in \mathcal{C}\left(\mathbb{R}^{+} \times \mathcal{P} \times E, \mathcal{P}\right)$ be such that

$$
\|f(t, x, y)\| \leq a_{0}(t)+a_{1}(t)\|x\|^{p}+a_{2}(t)\|y\|^{q}
$$

for any $(t, x, y) \in \mathbb{R}^{+} \times \mathcal{P} \times E$, where $a_{i} \in \mathcal{C}\left(\mathbb{R}^{+}, \mathbb{R}^{+}\right), i \in\{0,1,2\}$,

$$
\int_{0}^{\infty} m(s) a_{0}(s) d s \leq B_{1}, \quad \int_{0}^{\infty} m(s) a_{1}(s) e^{w s p} d s \leq B_{2}, \quad \int_{0}^{\infty} m(s) a_{2}(s) e^{\omega s q} d s \leq B_{3} .
$$

Theorem 3.2. Assume (H1). Then the problem (1.1) has at least one positive solution $x$ in $\mathcal{K}$ such that

$$
\sup _{t \in \mathbb{R}^{+}}\left(\left(\|x(t)\|+\left\|x^{\prime}(t)\right\|\right) e^{-\omega t}\right) \leq R
$$

Proof. For $x \in X$ define the operators

$$
\begin{aligned}
& T x(t)=(1+\varepsilon) x(t) \\
& F x(t)=-\varepsilon \int_{0}^{\infty} G(t, s) m(s) f\left(s, x(s), x^{\prime}(s)\right) d s, \quad t \in(0, \infty) .
\end{aligned}
$$

1. Note that $T: \mathcal{K}_{R} \rightarrow X$ is an $(1+\varepsilon)$-expansive operator.

2. Now we will prove that the operator $F: \overline{\mathcal{K}_{R}} \rightarrow X$ is continuous. From the assumption $(H 1)$, we can show that

$$
\sup _{t \in \mathbb{R}^{+}} e^{-\omega t}\left(\|F x(t)\|+\left\|(F x)^{\prime}(t)\right\|\right)<\infty,
$$

which imply that

$$
F\left(\overline{\mathcal{K}_{R}}\right) \subset X
$$

Let $\left\{x_{n}\right\}_{n \in \mathbb{N}},\{x\} \subset \overline{\mathcal{K}_{R}}$ with $\left\|x_{n}-x\right\|_{w} \rightarrow 0$, as $n \rightarrow \infty$. Hence, $\left\{x_{n}\right\}_{n \in \mathbb{N}}$ is bounded in $\overline{\mathcal{K}_{R}}$. Then there exists a positive constant $r$ such that $\max \left\{\left\|x_{n}\right\|_{\omega}, n \in \mathbb{N},\|x\|_{\omega}\right\} \leq r$. We have

$$
\begin{aligned}
\int_{0}^{\infty} & e^{-\omega t} G(t, s) m(s)\left\|f\left(s, x_{n}(s), x_{n}^{\prime}(s)\right)-f\left(s, x(s), x^{\prime}(s)\right)\right\| d s \\
\leq & \int_{0}^{\infty} e^{-\omega t} G(t, s) m(s)\left(\left\|f\left(s, x_{n}(s), x_{n}^{\prime}(s)\right)\right\|+\left\|f\left(s, x(s), x^{\prime}(s)\right)\right\|\right) d s \\
\leq & \int_{0}^{\infty} e^{-\omega t} G(t, s) m(s)\left(2 a_{0}(s)+a_{1}(s)\left(\left\|x_{n}(s)\right\|^{p}+\|x(s)\|^{p}\right)\right. \\
& \left.+a_{2}(s)\left(\left\|x_{n}^{\prime}(s)\right\|^{q}+\left\|x^{\prime}(s)\right\|^{q}\right)\right) d s \\
\leq & 2 B_{1} A+A B_{2}\left(\left\|x_{n}\right\|_{\omega}^{p}+\|x\|_{\omega}^{p}\right)+A B_{3}\left(\left\|x_{n}\right\|_{\omega}^{q}+\|x\|_{\omega}^{q}\right) \\
\leq & 2 A\left(B_{1}+B_{2} r^{p}+B_{3} r^{q}\right), \quad t \in(0, \infty),
\end{aligned}
$$


and

$$
\begin{aligned}
\int_{0}^{\infty} & e^{-\omega t}\left|G_{t}(t, s)\right| m(s)\left\|f\left(s, x_{n}(s), x_{n}^{\prime}(s)\right)-f\left(s, x(s), x^{\prime}(s)\right)\right\| d s \\
\leq & \int_{0}^{\infty} e^{-\omega t}\left|G_{t}(t, s)\right| m(s)\left(\left\|f\left(s, x_{n}(s), x_{n}^{\prime}(s)\right)\right\|+\left\|f\left(s, x(s), x^{\prime}(s)\right)\right\|\right) d s \\
\leq & \int_{0}^{\infty} e^{-\omega t}\left|G_{t}(t, s)\right| m(s)\left(2 a_{0}(s)+a_{1}(s)\left(\left\|x_{n}(s)\right\|^{p}+\|x(s)\|^{p}\right)\right. \\
& \left.\quad+a_{2}(s)\left(\left\|x_{n}^{\prime}(s)\right\|^{q}+\left\|x^{\prime}(s)\right\|^{q}\right)\right) d s \\
\leq & 2 B_{1} A+A B_{2}\left(\left\|x_{n}\right\|_{\omega}^{p}+\|x\|_{\omega}^{p}\right)+A B_{3}\left(\left\|x_{n}\right\|_{\omega}^{q}+\|x\|_{\omega}^{q}\right) \\
\leq & 2 A\left(B_{1}+B_{2} r^{p}+B_{3} r^{q}\right), \quad t \in(0, \infty) .
\end{aligned}
$$

Thus, the Lebesgue dominated convergence theorem both with the continuity of $f$ imply

$$
\sup _{t \in \mathbb{R}^{+}}\left(e^{-\omega t}\left\|\left(F x_{n}\right)(t)-(F x)(t)\right\|\right) \rightarrow 0, \quad \text { as } \quad n \rightarrow \infty
$$

and

$$
\sup _{t \in \mathbb{R}^{+}}\left(e^{-\omega t}\left\|\left(F x_{n}\right)^{\prime}(t)-(F x)^{\prime}(t)\right\|\right) \rightarrow 0, \quad \text { as } \quad n \rightarrow \infty
$$

As a result,

$$
\left\|F x_{n}-F x\right\|_{\omega} \rightarrow 0, \quad \text { as } \quad n \rightarrow \infty,
$$

i.e., the operator $F$ is continuous.

3. We have $F: \overline{\mathcal{K}}_{R} \rightarrow X$ and for $x \in \overline{\mathcal{K}}_{R}$ we get

$$
\begin{aligned}
\left(\|F x(t)\|+\left\|(F x)^{\prime}(t)\right\|\right) e^{-\omega t} \\
\quad \leq \varepsilon e^{-\omega t} \int_{0}^{\infty}\left(G(t, s)+\left|G_{t}(t, s)\right|\right) m(s)\left\|f\left(s, x(s), x^{\prime}(s)\right)\right\| d s \\
\leq \varepsilon e^{-\omega t} A \int_{0}^{\infty} m(s)\left(a_{0}(s)+a_{1}(s)\|x(s)\|^{p}+a_{2}(s)\left\|x^{\prime}(s)\right\|^{q}\right) d s \\
\leq \varepsilon e^{-\omega t} A \int_{0}^{\infty} m(s)\left(a_{0}(s)+a_{1}(s) e^{\omega p s} R^{p}+a_{2}(s) e^{\omega q s} R^{q}\right) d s \\
\leq \varepsilon e^{-\omega t} A\left(B_{1}+B_{2} R^{p}+B_{3} R^{q}\right) \\
\leq \varepsilon A\left(B_{1}+B_{2} R^{p}+B_{3} R^{q}\right) \\
\leq \tau \varepsilon \\
<\frac{\varepsilon}{4}, \quad t \in(0, \infty) .
\end{aligned}
$$

Hence,

$$
\|F x\|_{\omega} \leq \frac{\varepsilon}{4} .
$$

Therefore $F\left(\overline{\mathcal{K}_{R}}\right)$ is uniformly bounded. Since $F: \overline{\mathcal{K}_{R}} \rightarrow X$ is continuous, we have that $F\left(\overline{\mathcal{K}_{R}}\right)$ is equi-continuous. Consequently $F: \overline{\mathcal{K}_{R}} \rightarrow X$ is a 0 -set contraction. 
4. Let $y \in \overline{\mathcal{K}_{R}}$ be arbitrarily chosen. Set

$$
z(t)=\int_{0}^{\infty} G(t, s) m(s) f\left(s, y(s), y^{\prime}(s)\right) d s, \quad t \in(0, \infty) .
$$

We have that $z \in \mathcal{K}$ and using the above computations, we have

$$
\begin{aligned}
\left(\|z(t)\|+\left\|z^{\prime}(t)\right\|\right) e^{-\omega t} & \leq e^{-\omega t} \int_{0}^{\infty}\left(G(t, s)+\left|G_{t}(t, s)\right|\right) m(s)\left\|f\left(s, y(s), y^{\prime}(s)\right)\right\| d s \\
& \leq A\left(B_{1}+B_{2} R^{p}+B_{3} R^{q}\right) \\
& \leq A\left(B_{1}+B_{2} R^{p}+B_{3} R^{q}\right) \\
& \leq R,
\end{aligned}
$$

so, $\|z\|_{\omega} \leq R$. Therefore $z \in \mathcal{K}_{R}$. Also,

$$
\begin{aligned}
(I-T) z(t) & =-\varepsilon z(t) \\
& =-\varepsilon \int_{0}^{\infty} G(t, s) m(s) f\left(s, y(s), y^{\prime}(s)\right) d s \\
& =F y(t), \quad t \in(0, \infty) .
\end{aligned}
$$

Thus

$$
F\left(\overline{\mathcal{K}_{R}}\right) \subset(I-T)(\mathcal{K})
$$

5. Note that $0 \in \mathcal{K}_{R}$ and for any $x \in \partial \mathcal{K}_{R}$ we have

$$
\begin{aligned}
\|F x+T 0\|_{\omega} & =\|F x\|_{\omega} \\
& \leq \varepsilon A\left(B_{1}+B_{2} R^{p}+B_{3} R^{q}\right) \\
& \leq \varepsilon R \\
& =\varepsilon\|x\|_{\omega} .
\end{aligned}
$$

Assume that there exists an $x \in \partial \mathcal{K}_{R}$ such that

$$
F x+T x=x \text {. }
$$

Then

$$
\begin{aligned}
& \left(\|x(t)\|+\left\|x^{\prime}(t)\right\|\right) e^{-\omega t} \\
\geq \quad & (1+\varepsilon) e^{-\omega t}\left(\|x(t)\|+\left\|x^{\prime}(t)\right\|\right) \\
& -\varepsilon e^{-\omega t} \int_{0}^{\infty}\left(G(t, s)+\left|G_{t}(t, s)\right|\right) m(s)\left\|f\left(s, x(s), x^{\prime}(s)\right)\right\| d s \\
\geq \quad & (1+\varepsilon) e^{-\omega t}\left(\|x(t)\|+\left\|x^{\prime}(t)\right\|\right) \\
& -\varepsilon A\left(B_{1}+B_{2} R^{p}+B_{3} R^{q}\right), \quad t \in(0, \infty),
\end{aligned}
$$

or

$$
\varepsilon A\left(B_{1}+B_{2} R^{p}+B_{3} R^{q}\right) \geq \varepsilon e^{-\omega t}\left(\|x(t)\|+\left\|x^{\prime}(t)\right\|\right), \quad t \in(0, \infty),
$$

whereupon

$$
A\left(B_{1}+B_{2} R^{p}+B_{3} R^{q}\right) \geq R
$$

This is a contradiction.

By 1, 2, 3, 4, 5 and Lemma 2.6, we conclude that the operator $T+F$ has a fixed point $x \in \mathcal{K}_{R}$, which is a solution of the problem (1.1). This completes the proof. 
Remark 3.3. A discussion on the existence of a positive real $\mathrm{R}$ that verifies an inequality of the type $A\left(B_{1}+B_{2} R^{p}+B_{3} R^{q}\right)<R$, with respect to $\mathrm{p}$ and $\mathrm{q}$, is given in $[7$, Remark 3.2]. So that the same constant $\mathrm{R}$ checks the inequality $A\left(B_{1}+B_{2} R^{p}+B_{3} R^{q}\right)<\tau<\frac{1}{4}$ it is necessary that $A B_{1}<\tau$.

In the case when the last inequality does not hold, or in a general way the inequality $A\left(B_{1}+B_{2} R^{p}+B_{3} R^{q}\right)<\tau<\frac{1}{4}$ is not satisfied, additional conditions on the nonlinearity $f$ are needed to show that the operator $F$ is a $\ell$-set contraction.

To overcome the problem pointed out in the remark we consider the conditions (H2) and (H3).

(H2). For every $r>0$ and all subinterval $[a, b] \subset \mathbb{R}^{+}$, the nonlinearity $f$ is uniformly continuous on $[a, b] \times B_{E}(0, r) \times B_{E}(0, r)$, where $B_{E}(0, r)=\{x \in E:\|x\| \leq r\}$. (H3). There exist a positive functions $l_{1}, l_{2} \in L^{1}\left(\mathbb{R}^{+}\right)$such that

$$
\alpha\left(f\left(t, B_{1}, B_{2}\right)\right) \leq l_{1}(t) \alpha\left(B_{1}\right)+l_{2}(t) \alpha\left(B_{2}\right), \quad t \in \mathbb{R}^{+},
$$

for every bounded subsets $B_{1}, B_{2} \subset E$, where

$$
A \int_{0}^{+\infty} m(t)\left(l_{1}(t)+l_{2}(t)\right) d t<1
$$

And we present the following theorem.

Theorem 3.4. Assume $(H 1)-(H 3)$. Then the problem (1.1) has at least one positive solution $x$ in $\mathcal{K}$ such that

$$
\sup _{t \in \mathbb{R}^{+}}\left(\left(\|x(t)\|+\left\|x^{\prime}(t)\right\|\right) e^{-\omega t}\right) \leq R .
$$

Proof. The proof of this theorem is similar to that of Theorem 3.2, we will only show how the operator $F$ is a $\ell$-set contraction with $\ell<\varepsilon$ under conditions $(H 2)$ and $(H 3)$. Firstly, using Lemma 3.1 for $p(t)=e^{-\omega t}$ and $q(t)=e^{-\mu t}$ with $\mu<\omega$, we get the following result.

Lemma 3.5. Assume that $(H 1)$ holds. If $V$ be a bounded subset of $\overline{\mathcal{K}_{R}}$, then

$$
\alpha_{X}(F V)=\max \left(\sup _{t \in \mathbb{R}^{+}} \alpha_{E}\left(e^{-\omega t} F V(t)\right), \sup _{t \in \mathbb{R}^{+}} \alpha_{E}\left(e^{-\omega t}(F V)^{\prime}(t)\right)\right) .
$$

Proof. Let $V \subset \overline{\mathcal{K}_{R}}$ be arbitrary.

(a) $F(V) \subset X$ is a uniformly bounded set with respect to the norm $\|\cdot\|_{\mu}$. Indeed, as in Theorem 3.2, we obtain

$$
\|x\|_{\mu} \leq \varepsilon A\left(B_{1}+B_{2}\|x\|_{\mu}^{p}+B_{3}\|x\|_{\mu}^{q}\right), \forall x \in V .
$$

(b) The families $\left\{e^{-\omega t}(F V(t))\right\}_{t \in \mathbb{R}^{+}}$and $\left.\left\{e^{-\omega t}(F V)^{\prime}(t)\right)\right\}_{t \in \mathbb{R}^{+}}$are almost equicontinuous on $\mathbb{R}^{+}$. The proof is similar to the one in [5, Lemma 1.3.3].

Now, Suppose that $V \subset \overline{\mathcal{K}_{R}}$; we prove that there exists a constant $0 \leq \ell<\varepsilon$ such that $\alpha_{X}(F V) \leq \ell \alpha_{X}(V)$. Lemma 3.5 tells us that it is enough to verify that

$$
\max \left(\sup _{t \in \mathbb{R}^{+}} \alpha_{E}\left(e^{-\omega t} F V(t)\right), \sup _{t \in \mathbb{R}^{+}} \alpha_{E}\left(e^{-\omega t}(F V)^{\prime}(t)\right)\right) \leq \ell \alpha_{X}(V) \text {. }
$$


Let $x \in V$. we introduce, for each $n \geq 1$, the approximating operator $F_{n}$ by

$$
F_{n} x(t)=-\varepsilon \int_{0}^{n} G(t, s) m(s) f\left(s, x(s), x^{\prime}(s)\right) d s .
$$

Step 1. From $(H 1)$ and $(H 3)$, for every $t \in(0, \infty)$, we have that

$$
\begin{aligned}
& e^{-\omega t}\left\|F x(t)-F_{n} x(t)\right\| \\
\leq & \int_{n}^{+\infty} e^{-\omega t} G(t, s) m(s)\left\|f\left(s, x(s), x^{\prime}(s)\right)\right\| d s \\
\leq & \varepsilon A\left(\int_{n}^{+\infty} m(s) a_{0}(s) d s+\|x\|_{\omega}^{p} \int_{n}^{+\infty} e^{p \omega s} m(s) a_{1}(s) d s\right. \\
& \left.+\|x\|_{\omega}^{q} \int_{n}^{+\infty} e^{q \omega s} m(s) a_{2}(s) d s\right) .
\end{aligned}
$$

Similarly, we also have

$$
\begin{aligned}
& e^{-\omega t}\left\|\left(F_{n} x\right)^{\prime}(t)-(F x)^{\prime}(t)\right\| \\
\leq \quad & \varepsilon A\left(\int_{n}^{+\infty} m(s) a_{0}(s) d s+\|x\|_{\omega}^{p} \int_{n}^{+\infty} e^{p \omega s} m(s) a_{1}(s) d s\right. \\
& \left.+\|x\|_{\omega}^{q} \int_{n}^{+\infty} e^{q \omega s} m(s) a_{2}(s) d s\right) .
\end{aligned}
$$

As a consequence, we get

$$
\begin{aligned}
& \left\|F x-F_{n} x\right\|_{\omega} \\
= & \sup _{t \in \mathbb{R}^{+}}\left\{e^{-\omega t}\left(\left\|F x(t)-F_{n} x(t)\right\|+\left\|\left(F_{n} x\right)^{\prime}(t)-(F x)^{\prime}(t)\right\|\right)\right\} \\
\leq \quad & 2 \varepsilon A\left(\int_{n}^{+\infty} m(s) a_{0}(s) d s+\|x\|_{\omega}^{p} \int_{n}^{+\infty} e^{p \omega s} m(s) a_{1}(s) d s\right. \\
& \left.+\|x\|_{\omega}^{q} \int_{n}^{+\infty} e^{q \omega s} m(s) a_{2}(s) d s\right) .
\end{aligned}
$$

The convergence of the integrals guarantee that

$$
\begin{aligned}
& \lim _{n \rightarrow+\infty} \int_{n}^{+\infty} m(s) a_{0}(s) d s=0, \\
& \lim _{n \rightarrow+\infty} \int_{n}^{+\infty} e^{p \omega s} m(s) a_{1}(s) d s=0, \\
& \lim _{n \rightarrow+\infty} \int_{n}^{+\infty} e^{q \omega s} m(s) a_{2}(s) d s=0 .
\end{aligned}
$$

Then, for all $x \in V$ and $t \in(0, \infty)$, we have

$$
\begin{aligned}
& d\left(e^{-\omega t}\left(F_{n} x\right)(t), e^{-\omega t}(F V)(t)\right) \\
= & \inf _{y \in B}\left\{e^{-\omega t}\left(\left\|F_{n} x(t)-F y(t)\right\|+\left\|\left(F_{n} x\right)^{\prime}(t)-(F x)^{\prime}(t)\right\|\right)\right\} \\
\leq & e^{-\omega t}\left(\left\|F_{n} x(t)-F x(t)\right\|+\left\|\left(F_{n} x\right)^{\prime}(t)-(F x)^{\prime}(t)\right\|\right) \\
& \rightarrow 0, \text { as } n \rightarrow \infty,
\end{aligned}
$$

hence for every $t \in(0, \infty)$

$$
\sup _{x \in V} d\left(e^{-\omega t}\left(F_{n} x\right)(t), e^{-\omega t}(F V)(t)\right) \rightarrow 0 \text {, as } n \rightarrow \infty .
$$

Similarly, for every $t \in(0, \infty)$

$$
\sup _{x \in V} d\left(e^{-\omega t}\left(F_{n} V\right)(t), e^{-\omega t}(F x)(t)\right) \rightarrow 0, \text { as } n \rightarrow \infty .
$$

Then the Hausdorff distance

$$
H_{d}\left(e^{-\omega t} F V(t), e^{-\omega t} F_{n} V(t)\right)
$$


tends to 0 , as $n$ tends to $+\infty$ for all $t$ in $(0, \infty)$. The Lipschitz property of the MNC $\alpha$ guarantees

$$
\lim _{n \rightarrow+\infty} \alpha\left(e^{-\omega t} F_{n} V(t)\right)=\alpha\left(e^{-\omega t} F V(t)\right), \forall t \in(0,+\infty),
$$

and

$$
\lim _{n \rightarrow+\infty} \alpha\left(e^{-\omega t}\left(F_{n} V\right)^{\prime}(t)\right)=\alpha\left(e^{-\omega t}(F V)^{\prime}(t)\right), \forall t \in(0,+\infty),
$$

Step 2. In what follows, we estimate $\alpha\left(e^{-\omega t} F_{n} V(t)\right)$. Using Assumption (H3), Lemma 3.1 and the properties of the Green function lead to estimations:

$$
\begin{aligned}
\alpha\left(e^{-\omega t} F V(t)\right) & =\lim _{n \rightarrow+\infty} \alpha\left(e^{-\omega t}\left(F_{n} V\right)(t)\right) \\
& =\varepsilon \lim _{n \rightarrow+\infty} \alpha\left(\left\{e^{-\omega t} \int_{0}^{n} G(t, s) m(s) f\left(s, x(s), x^{\prime}(s)\right) d s\right\}, x \in V\right) \\
& \leq \varepsilon A \lim _{n \rightarrow+\infty} \int_{0}^{n} m(s) \alpha\left(f\left(s, x(s), x^{\prime}(s)\right), x \in V\right) d s \\
& \leq \varepsilon A \lim _{n \rightarrow+\infty} \int_{0}^{n} m(s)\left(l_{1}(s) \alpha\left(e^{-\omega s} V(s)\right)+l_{2}(s) \alpha\left(e^{-\omega s} V^{\prime}(s)\right)\right) d s \\
& \leq \alpha_{X}(V) \varepsilon A \lim _{n \rightarrow+\infty} \int_{0}^{n} m(s)\left(l_{1}(s)+l_{2}(s)\right) d s \\
& \leq \alpha_{X}(V) \varepsilon A \int_{0}^{+\infty} m(s)\left(l_{1}(s)+l_{2}(s)\right) d s .
\end{aligned}
$$

Since $t$ is arbitrary

$$
\sup _{t \in \mathbb{R}^{+}} \alpha\left(e^{-\omega t}(F V)(t)\right) \leq \ell \alpha_{X}(V)
$$

Similarly, we find that

$$
\sup _{t \in \mathbb{R}^{+}} \alpha\left(e^{-\omega t}(F V)^{\prime}(t)\right) \leq \ell \alpha_{X}(V),
$$

where, $\ell=\varepsilon A \int_{0}^{+\infty} m(s)\left(l_{1}(s)+l_{2}(s)\right) d s$.

From Lemma 3.5, we immediately deduce that

$$
\alpha_{X}(F V) \leq \ell \alpha_{X}(V)
$$

meaning that $F: \overline{\mathcal{K}_{R}} \rightarrow X$ is a $\ell$-set contraction with $\ell<\varepsilon$.

\section{An example}

Consider the following nonlinear boundary value problem for system of $n$ scalar differential equations in the Banach space $E=\mathbb{R}^{n}$ with the Euclidean norm

$$
\|x\|=\left(\sum_{i=1}^{n} x_{i}^{2}\right)^{\frac{1}{2}}
$$

with $x=\left(x_{1}, \ldots, x_{n}\right) \mid x_{i} \in \mathbb{R}, i=1, \ldots, n$ and let $0<p, q<1$ :

$$
\left\{\begin{array}{l}
-x_{i}^{\prime \prime}(t)+k^{2} x_{i}(t)=\frac{e^{-p \omega t}}{t^{2} \sqrt{t}} \frac{1-\cos t}{t+1}\left(1+\left(x_{i}(t)\right)^{p}+\left(x_{i}^{\prime}(t)\right)^{q}\right), t>0 \\
x_{i}(0)=0, \quad \lim _{t \rightarrow+\infty} x_{i}(t)=0, \quad i=1,2, \ldots, n .
\end{array}\right.
$$

Let $\mathcal{P}=\left\{x=\left(x_{1}, x_{2}, \ldots, x_{n}\right) \in \mathbb{R}^{n} \mid x_{i} \geq 0, i=1,2, \ldots, n\right\}$. Then $\mathcal{P}$ is a cone in $\mathbb{R}^{n}$ and clearly System (4.1) can be rewritten in the form (1.1) in E. In this case,

$$
x=\left(x_{1}, \ldots, x_{n}\right), y=\left(y_{1}, \ldots, y_{n}\right), \quad f=\left(f^{(1)}, \ldots, f^{(n)}\right)
$$


where for any $i \in\{1, \ldots, n\}, f^{(i)}$ is defined by

$$
f^{(i)}\left(t, x_{1}, \ldots, x_{n}, y_{1}, \ldots, y_{n}\right)=\frac{1-\cos t}{t+1}\left(1+x_{i}^{p}+y_{i}^{q}\right), \text { for } t \geq 0 .
$$

Then, we have $f^{(i)}$ is uniformly continuous on $[a, b] \times B_{E}(0, r)$, for all $[a, b] \subset I$ and $r>0$. The singular coefficient is given by $m(t)=\frac{e^{-p \omega t}}{t^{2} \sqrt{t}}$ for $t>0$. Then

$$
\begin{aligned}
\|f(t, x, y)\|^{2} & =\sum_{i=1}^{n}\left(f^{(i)}(t, x, y)\right)^{2} \\
& \leq 4 \frac{(1-\cos t)^{2}}{(t+1)^{2}}\left(n+\sum_{i=1}^{n} x_{i}^{2 p}+\sum_{i=1}^{n} y_{i}^{2 q}\right) \\
& \leq 4 \frac{(1-\cos t)^{2}}{(t+1)^{2}}\left(n+\left(\sum_{i=1}^{n} x_{i}^{2}\right)^{p}+\left(\sum_{i=1}^{n} y_{i}^{2}\right)^{q}\right) \\
& \leq 4 \frac{(1-\cos t)^{2}}{(t+1)^{2}}\left(n+\|x\|^{2 p}+\|y\|^{2 q}\right) .
\end{aligned}
$$

Hence,

$$
\|f(t, x, y)\| \leq a_{1}(t)\|x\|^{p}+a_{2}(t)\|y\|^{q}+a_{0}(t),
$$

where $a_{1}(t)=a_{2}(t)=2 \frac{1-\cos t}{t+1}$ and $a_{2}(t)=2 \sqrt{n} \frac{1-\cos t}{t+1}$.

Moreover, since in the vicinity the origin, $\frac{1-\cos s}{s^{2} \sqrt{s}} \sim \frac{1}{2 \sqrt{s}}$ and for any $\alpha>0$,

$$
\int_{0}^{+\infty} \frac{e^{-\alpha s}}{(s+1) \sqrt{s}} d x<\infty
$$

we deduce the convergence of the integrals

$$
\begin{gathered}
\int_{0}^{+\infty} e^{p \omega s} m(s) a_{1}(s) d s=2 \int_{0}^{+\infty} \frac{1-\cos s}{s^{2} \sqrt{s}(s+1)} d s \\
\int_{0}^{+\infty} m(s) a_{0}(s) d s=2 \sqrt{n} \int_{0}^{+\infty} \frac{(1-\cos s) e^{-p \omega s}}{s^{2} \sqrt{s}(s+1)} d s .
\end{gathered}
$$

Also, the integral

$$
\int_{0}^{+\infty} e^{q \omega s} m(s) a_{2}(s) d s=2 \int_{0}^{+\infty} \frac{(1-\cos s) e^{(q-p) \omega s}}{s^{2} \sqrt{s}(s+1)} d s
$$

is converge provided $p>q$.

Here the real numbers $p, q$ satisfy $0<p, q<1$, then there exists $R>0$ such that $A\left(B_{1}+B_{2} R^{p}+B_{3} R^{q}\right)<R$ (see [7, Remark 3.2]).

Finally, for every bounded subsets $D_{1}, D_{2} \subset E$ and for all $t \in \mathbb{R}^{+}, x \in D_{1}, y \in D_{2}$, we have

$$
\|f(t, x, y)\| \leq 2 \frac{1-\cos t}{t+1}\left(n+\|x\|^{2 p}+\|y\|^{2 q}\right) \leq 4\left(n+\|x\|^{2 p}+\|y\|^{2 q}\right) .
$$

Moreover, for all $0<t_{1}<t_{2}<+\infty, x \in D_{1}$, and $y \in D_{2}$, we have

$$
\begin{aligned}
& \lim _{t_{1} \rightarrow t_{2}}\left|f^{(i)}\left(t_{1}, x, y\right)-f^{(i)}\left(t_{2}, x, y\right)\right| \\
\leq & \lim _{t_{1} \rightarrow t_{2}}\left|\frac{1-\cos t_{1}}{t_{1}+1}\left(1+x_{i}^{p}+y_{i}^{q}\right)-\frac{1-\cos t_{2}}{t_{2}+1}\left(1+x_{i}^{p}+y_{i}^{q}\right)\right| \\
\leq & \left.\lim _{t_{1} \rightarrow t_{2}}\left(1+\|x\|_{\infty}^{p}+\|x\|_{\infty}^{q}\right) \mid \frac{1-\cos t_{1}}{t_{1}+1}-\frac{1-\cos t_{2}}{t_{2}+1}\right) \mid=0, \forall i=1, \ldots, n .
\end{aligned}
$$


Then $\lim _{t_{1} \rightarrow t_{2}}\left\|f\left(t_{1}, x, y\right)-f\left(t_{2}, x, y\right)\right\|=0$ and

$$
\begin{aligned}
& \lim _{t \rightarrow+\infty}\left|f^{(i)}(t, x, y)-\lim _{s \rightarrow+\infty} f^{(i)}(s, x, y)\right| \\
\leq & \lim _{t \rightarrow+\infty}\left|\frac{1-\cos t}{t+1}\left(1+x_{i}^{p}+y_{i}^{q}\right)-0\right|=0, \forall i=1, \ldots, n .
\end{aligned}
$$

Hence, $\lim _{t \rightarrow+\infty}\left\|f^{(i)}(t, x, y)-\lim _{s \rightarrow+\infty} f^{(i)}(s, x, y)\right\|=0$.

As a consequence, Corduneanu's compactness criterion ([1], p. 62) ensures that $f\left(t, D_{1}, D_{2}\right)$ is relatively compact in $\mathbb{R}^{n}$. So, $\alpha\left(f\left(t, D_{1}, D_{2}\right)\right)=0$, for all $t \in \mathbb{R}^{+}$ and all bounded subset $D_{1}, D_{2} \subset E$.

Theorem 3.4 ensures the sub-linear singular problem (4.1) has a bounded positive solution for every constants $k$ and all $0<p, q<1$.

Acknowledgments. We would like to thank the anonymous referees for their careful reading and helpful suggestions which led to a substantial improvement of the original manuscript.

\section{References}

[1] Corduneanu, C., Integral Equations and Stability of Feedback Systems, Academic Press, New York, 1973.

[2] Deimling, K., Ordinary Differential Equations in Banach Spaces, Springer, Berlin, Germany, 1977.

[3] Deimling, K., Nonlinear Functional Analysis, Springer-Verlag, Berlin, Heidelberg, 1985.

[4] Djebali, S., Travelling wave solutions to a reaction-diffusion system arising in epidemiology, Nonlinear Anal. Real World Appl., 2(2001), no. 4, 417-442.

[5] Djebali, S., Madjidi, F., Mebarki, K., Existence results for singular boundary value problems on unbounded domains in Banach spaces, Mediterr. J. Math., 11(2014), 45-74.

[6] Djebali, S., Mebarki, K., Fixed point index theory for perturbation of expansive mappings by $k$-set contractions, Topol. Methods Nonlinear Anal., 54(2019), no. 2, 613-640.

[7] Djebali, S., Mebarki, K., On the singular generalized Fisher-like equation with derivative depending nonlinearity, Appl. Math. Comput., 205(2008), no. 1, 336-351.

[8] Djebali, S., Moussaoui, T., A class of second order bvps on infinite intervals, Electron. J. Qual. Theory Differ. Equ., 4(2006), 1-19.

[9] Djebali, S., Saifi, O., Baoqiang, Y., Positive solutions for singular BVPs on the positive half-Line arising from epidemiology and combustion theory, Acta Math. Sci. Ser. B, 32(2012), no. 2, 672-694.

[10] Guo, D., Cho, Y.I., Zhu, J., Partial Ordering Methods in Nonlinear Problems, Shangdon Science and Technology Publishing Press, Shangdon, 1985.

[11] Guo, D., Lakshmikantham, V., Nonlinear Problems in Abstract Cones, Notes and Reports in Mathematics in Science and Engineering, vol. 5, Academic Press, Boston, Mass, USA, 1988.

[12] Guo, D., Lakshmikantham, V., Liu, X., Nonlinear Integral Equations in Abstract Spaces, Math. and Appl., vol. 373, Kluwer Academic Publishers, Dordrecht, The Netherlands, 1996. 
[13] Kneser, A., Untersuchung und asymptotische darstellung der integrale gewisser differentialgleichungen bei grossen werthen des arguments, J. Reine Angew. Math., 116(1896), 178-212.

[14] Lakshmikantham, V., Leela, S., Nonlinear Differential Equations in Abstract Spaces, Pergamon, Oxford, UK, 1981.

[15] Li, P., Chen, H., Wu, Y., Existence of solutions of $n$-point boundary value problems on the half-line in Banach spaces, Acta Appl. Math., 110(2010), 785-795.

[16] Liu, Y., Boundary value problems for second order differential equations on unbounded domains in a Banach space, Appl. Math. Comput., 135(2003), 569-583.

[17] Murray, J.D., Mathematical Biology, Biomathematics Texts, Springer-Verlag, 1989.

[18] Potter, A.J.B., A fixed point theorem for positive $k$-set contractions, Proc. Edinb. Math. Soc., 19(1974), 93-102.

[19] Zeidler, E., Nonlinear Functional Analysis and its Applications, Vol. I: Fixed Point Theorems, Springer-Verlag, New York, 1986.

Lyna Benzenati

Abderrahmane Mira University,

Laboratory of Applied Mathematics,

Faculty of Exact Sciences,

6000 Bejaia, Algeria

e-mail: benzenatilyna@gmail.com

Svetlin Georgiev Georgiev

Sofia University,

Faculty of Mathematics and Informatics,

Sofia, Bulgaria

e-mail: svetlingeorgiev1@gmail.com

Karima Mebarki

Abderrahmane Mira University,

Laboratory of Applied Mathematics,

Faculty of Exact Sciences,

6000 Bejaia, Algeria

e-mail: mebarqi_karima@hotmail.fr 